\title{
ANALYSIS OF HUMAN NEGOTIATIONS FOR e-BUSINESS IMPROVEMENTS
}

\author{
Beata Krawczyk-Brylka \\ Michal Piotrowski \\ Gdansk University of Technology \\ Faculty of Management and Economics \\ Faculty of Electronics, Telecommunications and Informatics \\ bkrawczy@zie.pg.gda.pl \\ bastian@eti.pg.gda.pl \\ POLAND
}

\begin{abstract}
Nowadays, we can abserve the transition of traditional business to electronic one, where negotiations still play very important role. In the paper we propose a general model of human negotiations and on the basis of which we have created a simplified model of quality analysis. The proposed quality model allows us to define various negotiation scenarios and to evaluate such negotiation metrics as completeness, effectiveness, performance and satisfaction. Two kinds of negotiations (f2f and chat) have been compared for buying/selling scenarios. More than 150 experiments have been carried out taking into account negotiation quality. Firstly, analysis has been made to examine the impact of negotiator personalities, experiences, roles and positions on that quality. Secondly, further studies have shown how negotiation quality is influenced by various types of negotiation strategies.
\end{abstract}

\section{INTRODUCTION}

In the time of transition of traditional to electronic business (e-business) companies should redesign their business processes in order to better satisfy customer needs (Patric, 2004). For this reason profound knowledge of such processes and market requirements should be acquired and deeply analysed (Peterson, 1997). Many researches based on specially defined questionnaires make a large-scale study to access e-business drivers facilitating achieving high levels of operational performance. These studies identify the critical links between e-business drivers, financial indicators and operational excellence measures (Barna, 2000). Besides, several descriptive theories and models try to describe selling/buying processes and improve negotiation procedures involved in such processes (Berenicke, 2003). In general, a buying/selling transaction comprises by the following six fundamental stages:

1. Identification of the user needs and recognition of the buyer motivations for buying a product.

2. Product brokering and information retrieval for consideration of different buying alternatives.

\footnotetext{
" This work was supported under national KBN grant No 4T11C00525
} 
3. Merchant brokering and first choice of the best alternatives using such buyer-provided criteria as prices, warranty periods, type of payments, delivery options, service availability, producer reputation, product quality.

4. Direct negotiation of different aspects including the above criteria to make selling/buying transaction accepted for both sides.

5. Contract definition for purchase, delivery and maintenance to finalise the negotiated transaction.

6. Past-purchase product life-phases and evaluation of user acceptation.

In practice, the above stages can overlap and migrate from one to another and can be implemented in different ways (Nguyen, 2004). One implementation technique is filtering (steps $1,2,3$ ) the attractive products by their features described on the Web. Another one is collaborative filtering which tries to recommend products based on various user options and ranking of their alternatives. Besides, data mining techniques (Adamavicius, 2001) can be used to discover the best patterns in customer purchasing and to exploit these patterns to help in taking decisions by buyers (steps $4,5,6$ ). In many cases agent based technologies can be used to support all stages of the buying process.

As it was shown in the stages given above, most business transactions involve negotiation procedures, which make use of different negotiation strategies and protocols (Maes, 1999). We distinguish two main kinds of negotiations: face to face (f2f) and computer mediated $(\mathrm{cm})$ ones. The former occurs in natural communication environment, where people meet and make conversation, the latter takes place when different telecommunications means are used. The simplest example of $\mathrm{cm}$ negotiations is negotiations via e-mail or chat (Picard, 2002). In the case of more advanced technologies people can create agents, which seek out potential buyers or sellers and negotiate with them on behalf of their owners. In our opinion proper implementation of agent to agent (a2a) or $\mathrm{cm}$ negotiation strategies requires good understanding of $\mathrm{f} 2 \mathrm{f}$ negotiations, which enables transforming traditional business into e-business more easily.

The paper focuses on $\mathrm{f} 2 \mathrm{f}$ negotiations. The general model is formalised in Section 2. In Section 3, quality attributes of negotiations are proposed and the way of estimating completeness, effectiveness, efficiency and satisfaction of negotiations is discussed. These attributes are strictly related to negotiation outcomes and therefore play important role in improving of e-business activities. The main experiments are described in Section 4. They focus on the representative selling/buying negotiation tasks showing the importance of negotiators' personalities, experience, roles, positions and negotiation strategies when referred to negotiation quality attributes. In Section 5 general remarks about e-business improvements and new open problems are given.

\section{MODEL OF NEGOTIATION}

Negotiation is the process that occurs between at least two corresponding parties (negotiators) and involves a certain subject (buying goods/products, taking decisions, preparing solutions, executing services). In general, this process can be described by a sixth tuple, as follows:

$$
\mathrm{N}=\langle\mathrm{S}, \mathrm{P}, \mathrm{G}, \mathrm{D}, \mathrm{C}, \mathrm{E}\rangle
$$


where:

$S$ is a subject of negotiation, as it was presented above;

$\mathrm{P}$ is a set of parties participating in negotiations; $\mathrm{P}=\left\{\mathrm{P}_{1}, \mathrm{P}_{2}, . ., \mathrm{P}_{\mathrm{i}} \ldots, \mathrm{P}_{1}\right\}$

The cardinality of $P$ is at least two i.e. $I \geq 2$, and each $P_{i}(i=1,2, \ldots, I)$ includes at least one negotiator. The parties first verbalise contradictionary demands and then move towards agreement by a search for new alternatives.

$\mathrm{G}$ is a set of goals describing attributes of the subject being under negotiation, i.e. $G=\left\{g_{1}, g_{2}, \ldots, g_{j} \ldots, g_{J}\right\}$, where $g_{j}(j=1,2, \ldots, J)$ presents one attribute, for instance in the case of selling/buying negotiations: product cost, delivery time, warranty conditions and etc. can be considered. The concrete value of $g_{j}$ is denoted by $v_{j}$.

$\mathrm{D}$ is a sequence of demands/replays formulated step by step by the parties during negotiation as modification of previous demands or presentation of new propositions, which should be discussed and modified later. $D=\left(D_{1}, D_{2}, \ldots, D_{k} \ldots\right.$, $D_{K}$ ), where: $K$ means the number of negotiations steps and $D_{k}$ is a value set of attributes: $D_{k}=\left\{v_{1}^{k}, v_{2}{ }^{k}, \ldots, v_{j}^{k} \ldots, v_{J}^{k}\right\}$. The set is a proposition of attribute values given by parties in $k$-th step of negotiation. $D_{k}$ represents a point in J-multiple area and sequence $D$ corresponds to the path of transitions from point $D_{1}$ to the point $D_{K}$. Such a graphical representation of negotiation steps in J-multiple area is called negotiation dance. For each party all values $v_{j}^{k}$ should satisfy the following acceptation condition:

$$
\min _{j}{ }^{k} \leq v_{j}{ }^{k} \leq \max _{j}{ }^{k} \text { for all } j, j=1,2, \ldots, J, k=1,2, \ldots, K
$$

$C$ denotes the contract for the subject, when all parties make acceptation for the demand formulated in $\mathrm{K}$-th step of negotiation. Then $\mathrm{D}_{\mathrm{K}}$ is called the outcome of negotiation (contract values), i.e.:

$$
\mathrm{O}=\mathrm{D}_{\mathrm{K}}=\left\{\mathrm{v}_{1}{ }^{\mathrm{K}}, \mathrm{v}_{2}{ }^{\mathrm{K}}, \ldots, \mathrm{v}_{\mathrm{j}}^{\mathrm{K}} \ldots, \mathrm{v}_{\mathrm{J}}{ }^{\mathrm{K}}\right\}=\left\{\mathrm{c}_{1}, \mathrm{c}_{2}, \ldots, \mathrm{c}_{\mathrm{j}} \ldots, \mathrm{c}_{\mathrm{J}}\right\}
$$

$E$ is an environment, where negotiation is being run. In the case of natural environment, $\mathrm{f} 2 \mathrm{f}$ negotiation takes place. Using communication via Internet we have chat negotiation, and using agent technology we organise negotiation in more automatic way (i.e. a2a negotiation or e-negotiation). E may play an essential role in achieving the required level of negotiation quality and in implementation of ebusiness activities.

Parties can formulate demands in different order and all demands can be presented by all parties either at once or sequentially one by one. There are no rules in which order demands should be presented. Moreover, parties can remain either passive (when they only accept or reject the demands made by others) or active (when they can change the current proposals to make them more likely to be accepted). Each simple change of demands means a new step of negotiation. If current demands are not acceptable for the other parties, these parties can return to the previous proposition which seems more promising. Therefore, the track of some propositions (D'CD) should be kept and recovery mechanisms should support such return operations. Taking into account a way of demand formulation and presentation, negotiation strategies can be competitive (the parties focus on the best 
outcomes for themselves), balanced (the parties are looking for a compromise following with the objective conditions) and collaborative (the parties try to understand reasoning of other sides). Negotiation strategies used by one party can vary over time according to the assumed tactics and current negotiator satisfactions and feelings. A graph of negotiation dance very well suggests types of negotiation strategies used for a contract completion.

\section{NEGOTIATION QUALITY ATTRIBUTES}

The assumed negotiation model allows us to define quality attributes of negotiation, as it is shown in Fig. 1a. Quality of negotiation (QoN) can be considered taking into account the main aspects: personal, process and technical ones. Correct description of subjects (QoS) and negotiation goals (QoG) and their proper understanding by the parties, as well as personality characteristics of the parties (QoP) are grouped as personal aspects of negotiation quality. On the other hand, processes of demands formulation (QoD) and contract preparation (QoC) belong to negotiation process (process aspects). Places and conditions of negotiations and technical means create negotiation environment (QoE), which is the other aspect of negotiation quality. In many papers, all these quality aspects are described and analysed separately. Moreover, the majority of the papers focus on the technical aspects only, primarily of how to improve communication channels for delivering the required information. Finally, the negotiation outcome (QoO) strictly depends on all aspects given above and directly determines a level of negotiation quality.

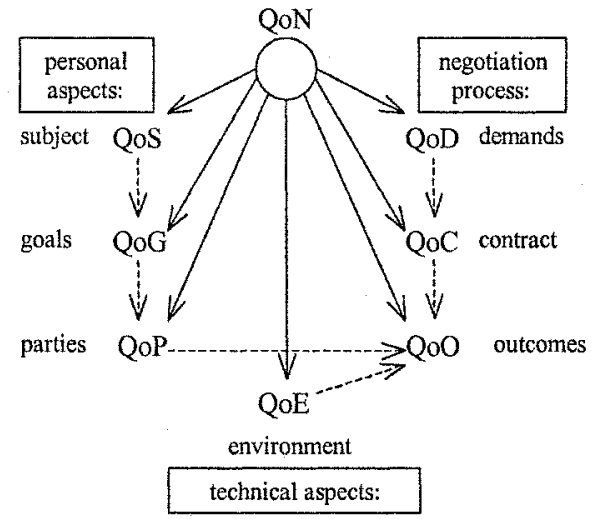

a)

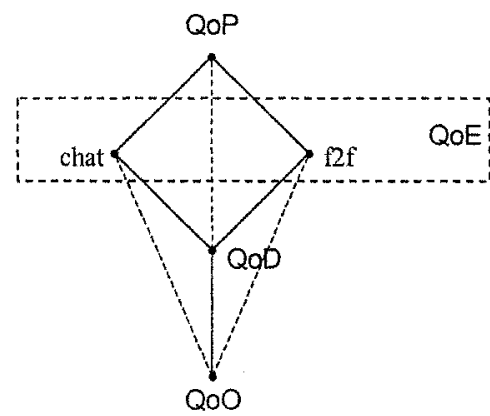

b)

Figure $1-$ a) The main attributes of negotiation strategy b) The simplified negotiation model

The paper represents the combined approach which takes into account all the presented attributes. However, to avoid huge complexity some simplifications have been made. First, we focused on personality attributes, which in a way represent other personal aspects. Second, we chose QoO as representative attribute of the contract, and the negotiation dance as the base for calculating QoD. Third, we 
limited technical aspects to two communication channels: $2 \mathrm{f}$ and chat. As a result we obtained the quality model as it is shown in Fig. $1 \mathrm{~b}$.

It has been assumed that the quality of negotiations is higher when negotiators consider all required goals and obtain satisfactory outcomes in a shorter time. As it was shown in Section 1, negotiations are the essential part of e-business and make clients more content because of a shorter time of execution of business transactions and due to better outcome included in the final contract.

Table 1 shows the main quality attributes and metrics taken into consideration in this paper. To present quantitative metrics we consider selling/buying negotiations for $\mathrm{I}=2$ with one negotiator in each party, and $\mathrm{J}=5$. Let assume that $\mathrm{b}$ and $\mathrm{s}$ represent buyer and seller respectively.

Table 1 - Main quality attributes and metrics for model shown in Fig. 1 b)

\begin{tabular}{|l|l|}
\hline \multicolumn{1}{|c|}{ Quality attributes } & \multicolumn{1}{c|}{ Quality metrics } \\
\hline Parties characteristics & $\begin{array}{l}\text { personality (personal feature of the negotiator } \\
\text { measured by sociology and psychology tests } \\
\text { experience (low, medium and high in business } \\
\text { activities) } \\
\text { role of participants (seller, buyer) } \\
\text { position in negotiation (measured by distance to } \\
\text { alternatives) }\end{array}$ \\
\hline Communication channels & $\begin{array}{l}\text { f2f, chat } \\
\text { competitive, competitive/balanced, balanced, } \\
\text { balanced/collaborative, collaborative }\end{array}$ \\
\hline Outcomes & $\begin{array}{l}\text { effectiveness - the percentage of negotiations } \\
\text { with complete contracts in the considered } \\
\text { experiment } \\
\text { performance - distribution of negotiation times } \\
\text { for complete contract in the considered } \\
\text { experiment } \\
\text { completeness - the percentage of goals taken into } \\
\text { consideration for each negotiation in the } \\
\text { considered experiment } \\
\text { satisfaction - the relative difference (in \%) } \\
\text { between the obtain outcome and the expected } \\
\text { outcomes }\end{array}$ \\
\hline
\end{tabular}

Fig. 2 defines main parameters used for analysing quality of negotiations. Symbols of $\min (x)$ and $\max (x)$ denote boundary values of the considered attribute $g$ for negotiator $\mathrm{x}$, where $\mathrm{x}$ represents either a buyer (b) or a seller (s). These values allow to estimate the most expecting outcome area (dotted rectangular area in Fig. 2) and to estimate the mean values of demand $g$ (i.e. $m v(x)$ ) for both negotiators, i.e.:

$$
m v(x)=0.5[\min (x)+\max (x)]
$$

In similar way we can estimate the balance outcome $(b v)$ :

$$
b v=0.5[\min (s)+\max (b)]
$$

The position of negotiator $\mathrm{x}$ before negotiation, denoted by $p o s(x)$, can be defined as difference in values between two parameters: $b v$ and $m v(x)$. Then $\operatorname{pos}(x)$ 
can be evaluated in the following way:

$$
\operatorname{pos}(x)=\left\{\begin{array}{c}
\text { low if } \Delta x>\beta \cdot b v \\
\text { medium if } \alpha \cdot b v<\Delta x<\beta \cdot b v \\
\text { high if } \Delta x<\alpha \cdot b v
\end{array}\right.
$$

where $\Delta x=|b \nu-m v(x)|$, coefficients $\alpha, \beta$ describe the boundary levels of position and in experiments we assume that $\alpha=0.1 \div 0.2$ and $\beta=2 \alpha$.

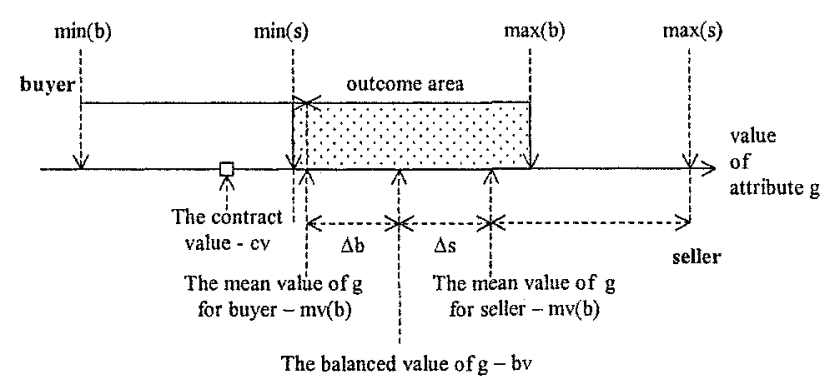

Figure 2 - Illustration of one of possible distribution of demands and outcomes for attribute $g$ of negotiation

Completeness of negotiation can be evaluated in the following way:

completeness $[\%]=(J * / J) \cdot 100 \%$,

where $J^{*}$ is the number of the subject attributes taken into consideration during negotiation.

Satisfaction of negotiation related to the contract value $(c v)$ is evaluated by each negotiator $\mathrm{x}$ separately. Let denote it by $\operatorname{sat}_{x}(c v)$, then in general it can be expressed by the following formula:

$$
\operatorname{sat}_{x}(c v)=\left\{\begin{array}{c}
0 \% \text { if } c v \leq \min (x) \\
w(x) \% \text { if } \min (x)<c v<\max (x) \\
100 \% \text { if } c v \geq \max (x)
\end{array}\right.
$$

where: $w(x)=[c v-\min (x)] /[\max (x)-\min (x)] \cdot 100$. However the above formula should be adjusted according to context of $g$.

Let note that in Fig. 2, for $g=$ warranty period $25 \% \leq s a t_{b}(c v) \leq 50 \%$ and $s a t_{s}(c v)=0 \%$, but for $g=$ price $50 \% \leq s a t_{b}(c v) \leq 75 \%, s a t_{s}(c v)=0 \%$.

We can normalise the result by restriction of satisfaction analysis to the existing outcome area, then $s a t_{b}(c v)=100 \%-s a t_{s}(c v)$.

All above formulas can be used for analysing different kinds of negotiations including $\mathrm{f} 2 \mathrm{f}$ and $\mathrm{cm}$ ones. If our experiments consist of many tours of negotiations, the completeness and satisfaction can be expressed as either distribution of values of such metrics or as their mean values. 


\section{EXPERIMENTAL RESULTS}

To analyse negotiation quality 156 selling/buying negotiation experiments have been done. Each experiment have been proceeding according to the scheme shown in Fig. 3. To implement this scheme, computer-based system GAJA was designed and implemented (Piotrowski, 2006). It is functional as it offers the possibilities to:

- monitor several kinds of negotiations (sell/buy, ranking, enterprise),

- define many versions (instances) of experiments for each kind of negotiations,

- support activities of different types of users (experts, negotiators, administrators),

- quality analysis of negotiators' behaviour, negotiation processes and negotiation outcomes.

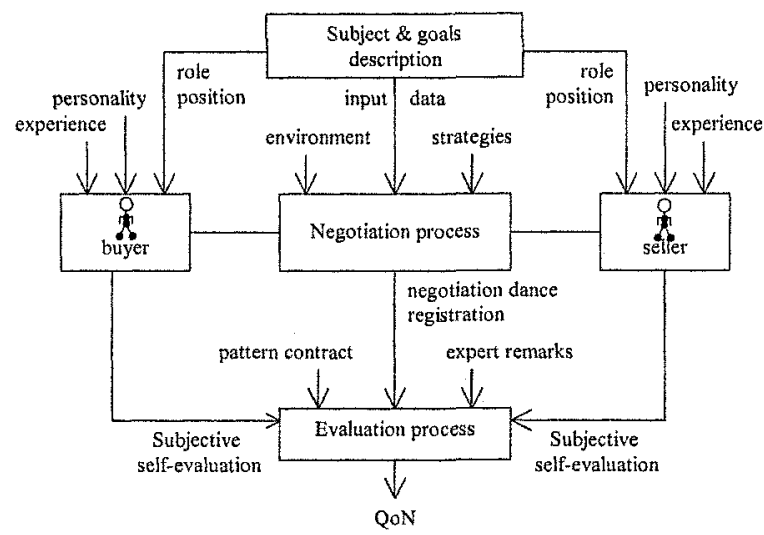

Figure 3 - Experiment schema for negotiation quality analysis

Table 2 shows the basic items of experiments. All negotiators were randomly divided into two groups: sellers and buyers. Then, their personality was tested, and pairs of negotiators (seller and buyer) were randomly assigned to one of two environments ( $\mathrm{f} 2 \mathrm{f}$ and chat). Next, each pair of negotiators received the same description of the negotiation task with some particular differences for seller and buyer. The same suitable initial conditions, admissible values of considered attributes were given for all participants.

After completion of a contract or after finishing negotiation without contract all negotiators made self-evaluation of both the processes and the obtained outcomes. Besides, the negotiation process was recorded as chat logs or video logs, which enables examining outcomes by experts. All collected data can be sent to STATISTICA application and analysed in many different ways.

Below we present only several experiment results strictly connected with negotiation quality analysis. Fig. 4 shows professional experiences of respondents. The biggest group (37\%) has been working in business for $1-5$ years. The next group $(25 \%)$ has less than 1 year experience, which means that respondents were students or last year's graduates of our university. 
Table 2 - Basic items of experiments

\begin{tabular}{|l|l|}
\hline \multicolumn{1}{|c|}{ Items } & \multicolumn{1}{c|}{ Descriptions } \\
\hline Negotiators & $\begin{array}{l}156 \text { pairs of negotiators, } 50 \% \text { female } 50 \% \text { male, } \mathrm{I}=2 \\
30 \% \text { for f2f negotiations, } 70 \% \text { for chat }\end{array}$ \\
\hline Roles, positions & given in advance in description of negotiation task \\
\hline Subjects & $\begin{array}{l}\text { selling/buying negotiation of medical equipment } \\
\text { having concrete functionality and quality }\end{array}$ \\
\hline Attributes & $\begin{array}{l}\text { price, warranty period, bank credit duration, delivery } \\
\text { time, cost of maintenance course, } \mathrm{J}=5\end{array}$ \\
\hline Negotiation strategies & not suggested \\
\hline Evaluations & $\begin{array}{l}\text { Special questionnaires to fulfil by experts or } \\
\text { negotiators }\end{array}$ \\
\hline
\end{tabular}

Other experimental results are shown in Fig. 5. It is easy to notice that chat negotiations take more time in comparison to $2 \mathrm{f}$ negotiations. Besides, performance depends also on the negotiator experience. Time of negotiation is shorter nearly twice for negotiators with 10-years experience in business compared to time of negotiation for negotiators with no or little experience. Results show that negotiators' personalities are also essential factors in this area.

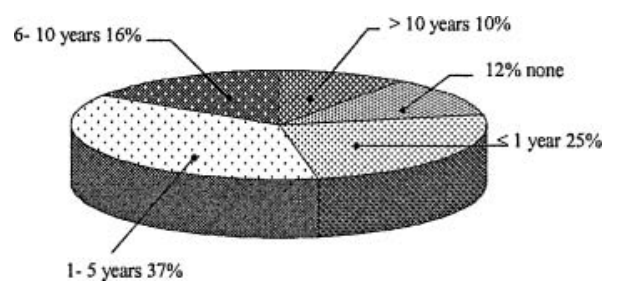

Figure 4-Professional experience of respondents

General effectiveness of negotiations was $97,1 \%$, but for $\mathrm{f} 2 \mathrm{f}$ and chat negotiations $98,4 \%$ and $96,6 \%$ respectively, which means that also $2 \mathrm{f}$ negotiations are a bit more effective, but the difference is not really big. General completeness of negotiations was $89,1 \%$, but for $\mathrm{f} 2 \mathrm{f}$ and chat negotiations $98,1 \%, 84,5 \%$ respectively, which means that there is more opportunity to lose some attributes in chat negotiations. The reason for ending $\mathrm{cm}$ negotiation before reaching contract is the tendency to use the strategy of testing partner motivation and position by expressing lack of approval for his demands. In context of a few non-verbal signals (that are important for building positive negotiation climate and avoiding misunderstandings) it can lead to incompleteness of negotiation.

Factor analysis shows that personal features are the most important factors for chat negotiation effectiveness. The most important personal features are conscientiousness and extraversion, high level of them indicates high motivation and high negotiation position. It is especially important for women negotiating via Internet, because to be effective they need to be more conscientious and much less agreeable than in $\mathrm{f} 2 \mathrm{f}$ meetings. 
The most important factor for $\mathrm{f} 2 \mathrm{f}$ negotiation is the subjective perception of negotiation process. The parties that estimate the negotiation climate as friendly are much more motivated to reach the compromise. The personal feature important for $\mathrm{f} 2 \mathrm{f}$ negotiators is neuroticism - the lower level better negotiation results.

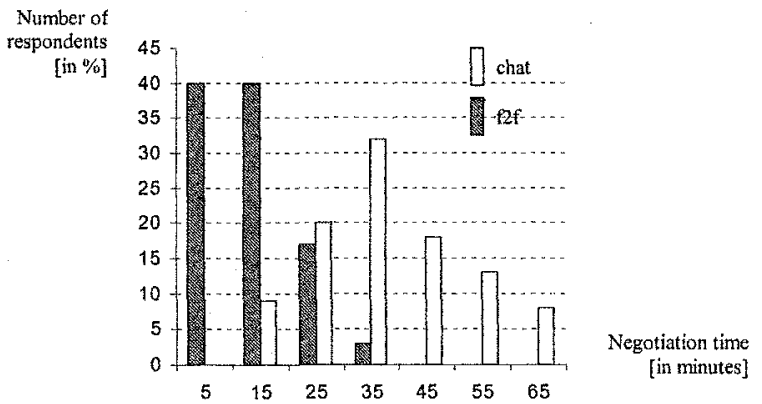

Figure 5 - Distribution of negotiation time (performance) for $\mathrm{f} f \mathrm{f}$ and chat channels

Fig. 6 shows that negotiators working in natural environment gain the biggest satisfaction. However, in the case of chat negotiations more respondents get the mean satisfaction.

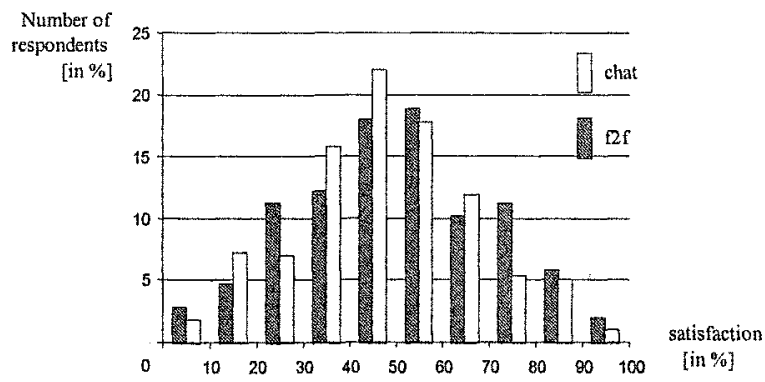

Figure 6 - Distribution of satisfaction for $\mathrm{f} 2 \mathrm{f}$ and chat negotiations

Fig. 7 shows that, in general, negotiators communicating by chat use the competitive strategy (they use strategies of accusing and frightening interlocutors more frequently), inversely than negotiators working in natural environment. The latter prefer the comoperative strategy. However, the balanced strategy for both environments is the most preferable one.

\section{FINAL CONCLUSIONS}

The paper presents a quantitative model of negotiation. This model enables evaluating quality of negotiation in different environments and comparing these environments in order to point out the most important drawbacks. Four quality attributes are defined and analysed. It was shown that the effectiveness and 
completeness are strictly related to negotiators' personality and experience. The performance and satisfaction depend on personality of negotiators and their positions (evidently in chat negotiations). Negotiation strategies also impact on the performance and satisfaction.

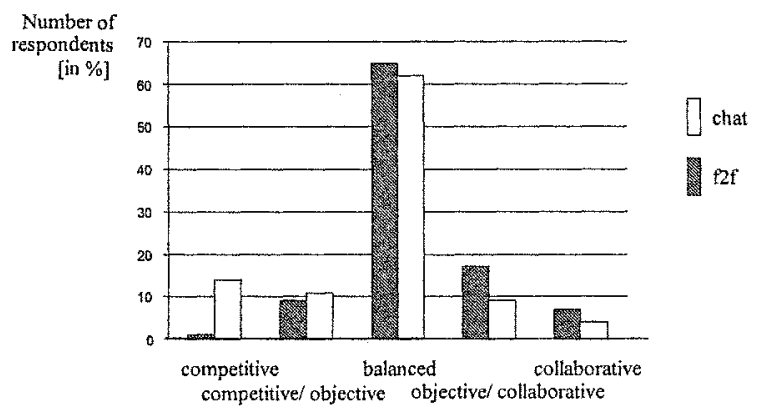

Figure 7 - Used negotiation strategies in $\mathrm{f} 2 \mathrm{f}$ and chat channels

A very important conclusion is that technical aspects are not the main ones but they are still very important for chat negotiations. This means that application of modern communication technology needs further changes to increase negotiation quality. To improve the completeness, effectiveness, performance and satisfaction of negotiations more intelligent and flexible tools are required. Such tools should be able to check, control and predict behaviour of negotiators and register, analyse and predict the negotiation dances. Then we will be able to control an increase of negotiation quality and in consequence to improve quality of e-business transactions.

\section{REFERENCES}

1. Adamavicius Gediminas, Tuzhilin Alexander. Using data mining methods to build customer profiles. IEEE Computer, Feb. 2001.

2. Barna Anitesh, Konana Prabhudev, Whinston Andrew, Yin Fang. Making e-business pay: Eight key drivers for operational success. IT Professional Nov./Dec. , 2000.

3. Berenicke Morcel, Langendorfer Peter. Towards automatic negotiation of privacy contracts for Internet services. Proc. of the 11 th Int. Conference on Networks, 2003.

4. Maes Pattie, Guttman RH, Munkas AG. Agent that Buy and Sell. Communication of the ACH, March, 1999.

5. Nguyen TD, Jennings NR. Coordinating multiple concurrent negotiations. Pwc. of AAHAS'04, New York, USA.

6. Patric Charles, Sahai Akhil. Business processes on the Web. TEEE Internet Computing, Jan./Feb. , 2004.

7. Peterson Erika, Thompson Leigh. Negotiation Teamwork: The impact of information distribution and accountability on performance depends on the relationship among team members. Organizational, Behavior and Human Decision processes, No3, 1997.

8. Picard Willy. Multi-facet analysis of e-negotiations PhD Thesis. Ecole Nationale Superieure des Telecommunications, Paris, 2002.

9. Piotrowski Michał, Krawczyk-Bryłka Beata. The Web Based System for Recording and Analysing Different Kinds of Negotiations: WEBIST 2006, Proceedings of the Second International Conference on Web Information Systems and Technologies, Society, e-Business and eGovernment/e-Learning, Portugal, 2006. 